04,06

\title{
Температурно-частотная зависимость диэлектрического отклика в мультиферроике $\mathrm{LuFe}_{2} \mathrm{O}_{4}$
}

\author{
() Г.М. Гаджиев ${ }^{1}$, А.Г. Гамзатов ${ }^{1, \uparrow, ~ Р . А . ~ А л и е в ~}{ }^{1}$, Н.С. Абакарова ${ }^{1}$, Л.Л. Эмирасланова ${ }^{2}$, \\ М.Н. Маркелова ${ }^{3}$, А.Р. Кауль ${ }^{3}$ \\ ${ }^{1}$ Институт фризики им. Х.И. Амирханова ДФИЦ РАН, \\ Махачкала, Россия \\ 2 Дагестанский государственный университет, \\ Махачкала, Россия \\ ${ }^{3}$ Московский государственный университет, \\ Москва, Россия \\ ฯ E-mail: gamzatov_adler@mail.ru
}

Поступила в Редакцию 30 декабря 2019 г.

В окончательной редакции 30 декабря 2019 г.

Принята к публикации 10 января 2020 г.

\begin{abstract}
Представлены результаты исследования температурно-частотных спектров комплексной диэлектрической проницаемости мультиферроика $\mathrm{LuFe}_{2} \mathrm{O}_{4}$ в области температур $100-400 \mathrm{~K}$ и частот $20 \mathrm{~Hz}-120 \mathrm{MHz}$. Обнаружены особенности в виде смены знака температурного коэффициента диэлектрической проницаемости, а также в виде максимумов и минимумов $a c$-проводимости в области температур $\sim 150-350 \mathrm{~K}$ и частот $\sim 30-120 \mathrm{MHz}$. Данные особенности обусловлены независимыми резонансными механизмами: совпадением частоты измерительного $a c$-напряжения с частотой перескоков электронов при реализации прыжковой проводимости; близостью периода измерительного сигнала к времени формирования барьерного слоя на границе контакта с образцом; приближением $а с$-частоты внешнего поля к частоте структурных элементов образца.
\end{abstract}

Ключевые слова: мультиферроик, импеданс, диэлектрическая проницаемость, $a c$-проводимость.

DOI: 10.21883/FTT.2020.05.49229.13M

\section{1. Введение}

Наблюдающийся в последние годы всплеск активности в исследовании мультиферроидных материалов связан с открытием в них магнитоэлектрического (МЭ) эффекта, который, в отличие от электродинамики Максвелла, подразумевает порождение намагниченности статическим электрическим полем и индуцирование электрической поляризации статическим магнитным полем [1]. Возможность проявления МЭ-эффекта в мультиферроиках при комнатной температуре представляет интерес с точки зрения их использования: в элементах памяти, магнитных сенсорах, емкостных электромагнитах и др. устройствах не предполагающие протекание постоянных электрических токов и сопряженных с этим тепловых потерь [2].

Одним из материалов, призванных демонстрировать мультиферроидные свойства является соединение феррит лютеция $\mathrm{LuFe}_{2} \mathrm{O}_{4}$ [3]. Этот материал имеет гексагональную слоистую структуру с пространственной группой $R \overline{3} m$. В такой структуре имеются двойные слои треугольных решеток атомов железа ( $W$-слои), которые содержат одинаковое число $\mathrm{Fe}^{+2}$ и $\mathrm{Fe}^{+3}$ ионов [3]. Возникновение электрической поляризации в данных материалах обусловлено процессами электронной корреляции и геометрической фрустрацией $\left(\mathrm{Fe}^{2+} / \mathrm{Fe}^{3+}\right)$ зарядов в области температур между 350 и $500 \mathrm{~K}$, приводящей к
2D-зарядовому упорядочению, а при температуре ниже $320 \mathrm{~K}$ - к 3D-зарядовому упорядочению. Возникающее в результате такого процесса 3D-зарядовое упорядочение, в свою очередь, индуцирует магнитное упорядочение при уменьшении температуры ниже $250 \mathrm{~K}$ [4,5]. Близость температуры зарядового и магнитного упорядочения к комнатной температуре, делает мультиферроик $\mathrm{LuFe}_{2} \mathrm{O}_{4}$ привлекательным материалом для применения в вышеуказанных приложениях.

Распространенным методом исследования диэлектрических свойств мультиферроиков является исследование температурной зависимости комплексной диэлектрической проницаемости - $\varepsilon(T)$. В большинстве работ, посвященных импедансной спектроскопии соединений $\mathrm{LuFe}_{2} \mathrm{O}_{4}$, измерения, в основном, были выполнены при частоте тестирующего переменного электрического поля от $20 \mathrm{~Hz}$ до $1 \mathrm{MHz}$ [2,6-10]. Практически отсутствуют работы, в которых обсуждается аномальное поведение $\varepsilon(T) \quad \mathrm{LuFe}_{2} \mathrm{O}_{4}$ в области температур $200-350 \mathrm{~K}$ при частотах измерительного сигнала несколько десятков мегагерц. В имеющихся единичных работах $[11,12]$ из-за большого шага по частоте область между 1 и $100 \mathrm{MHz}$ пропущена, поэтому авторы не заметили особенности $\varepsilon(T)$ в этой области. Результаты исследования температурной и частотной зависимости импеданса в системе $\mathrm{LuFe}_{2} \mathrm{O}_{4}$ вблизи температуры магнитного упорядочения $(\sim 250 \mathrm{~K})$ показали, что зависимости импе- 
данса $Z$ и угла сдвига фазы между током и напряжением в интервале частот $30-80 \mathrm{MHz}$ носит резонансный характер $[13,14]$. В данной работе нами проведен анализ реальной $\varepsilon^{\prime}$ и мнимой $\varepsilon^{\prime \prime}$ компоненты комплексной диэлектрической проницаемости, полученных обработкой данных из [13,14], которые также демонстрируют аномальное поведение в указанной выше температурной и частотной области.

В нашей работе использовались керамические образцы $\mathrm{LuFe}_{2} \mathrm{O}_{4}$ изготовленные по методике, описанной в [15]. Характеристики образцов и условия эксперимента соответствуют, описанным в работе [13].

\section{2. Экспериментальные результаты и их анализ}

На рис. 1 представлены температурные зависимости $\varepsilon(T)$ для различных частот ас-напряжения в области температур $100 \mathrm{~K}<T<400 \mathrm{~K}$. Кривые $\varepsilon^{\prime}=f(T)$, измеренные на частоте переменного напряжения $20 \mathrm{~Hz}, 5,30$, 35 и $45 \mathrm{MHz}$ характеризуются наличием острых минимумов. На $\varepsilon^{\prime}(T)$ для 100 и $120 \mathrm{MHz}$ наблюдаются незначительные провалы. Общий ход зависимости $\varepsilon^{\prime}(T)$, если не принимать во внимание минимумы, характеризуется положительным значением температурного коэффициента диэлектрической проницаемости - $\alpha_{\varepsilon}=1 / \varepsilon \cdot(d \varepsilon / d T)$ в интервале $\sim 200-300 \mathrm{~K}$ для частот переменного напряжения от $20 \mathrm{~Hz}$ до $5 \mathrm{MHz}$, как и сообщалось в предыдущих работах $[3,7,9,11]$. При повышении частоты $a c$-сигнала от $\sim 30$ до $60 \mathrm{MHz}$ кривые $\varepsilon^{\prime}(T)$ демонстрируют отрицательное значение $\alpha_{\varepsilon}$ в области $\sim 200-300$ то есть они имеют зеркально симметричный характер по отношению к кривым в верхней рамке (рис. 1). При дальнейшем увеличении частоты высокотемпературная полка $\varepsilon^{\prime}(T)(T>250 \mathrm{~K})$ располагается выше низкотемпературной $(T<250 \mathrm{~K})$ - кривые $\varepsilon^{\prime}(T)$ вновь приобретают вид, присущий для низких частот. Следует отметить, что $\varepsilon^{\prime}(T)$ для $30 \mathrm{MHz}$ практически не зависит от температуры за исключением области минимума. Дисперсия $\varepsilon^{\prime}$ в низкотемпературной и высокотемпературной области, в целом, подчиняется дебаевскому типу релаксации.

Из рис. 2 видно, что частота переменного напряжения очень сильно влияет на вид $\varepsilon(T)$ : модифицируя его от экспоненциальной зависимости для низких частот (вставка в верхней рамке на рис. 2) до ярко выраженных максимумов и минимумов $\sigma(T)$ для высоких частот. При увеличении частоты тестового сигнала до $500 \mathrm{kHz}$ на $\sigma(T)$ начинает проявляться слабый максимум на фоне экспоненциальной подставки, который при дальнейшем увеличении частоты до $35 \mathrm{MHz}$ оформляется в виде четкого горба. В области температур, где на $\varepsilon^{\prime}(T)$ наблюдаются минимумы, на соответствующих зависимостях $\sigma(T)$ имеются максимумы. Положения минимумов $\varepsilon^{\prime}(T)$ даже если строго не совпадают с максимумами $\sigma(T)$, все же находятся в пределах полуширины пика проводимости $\Delta T_{\sigma}$, определенной как
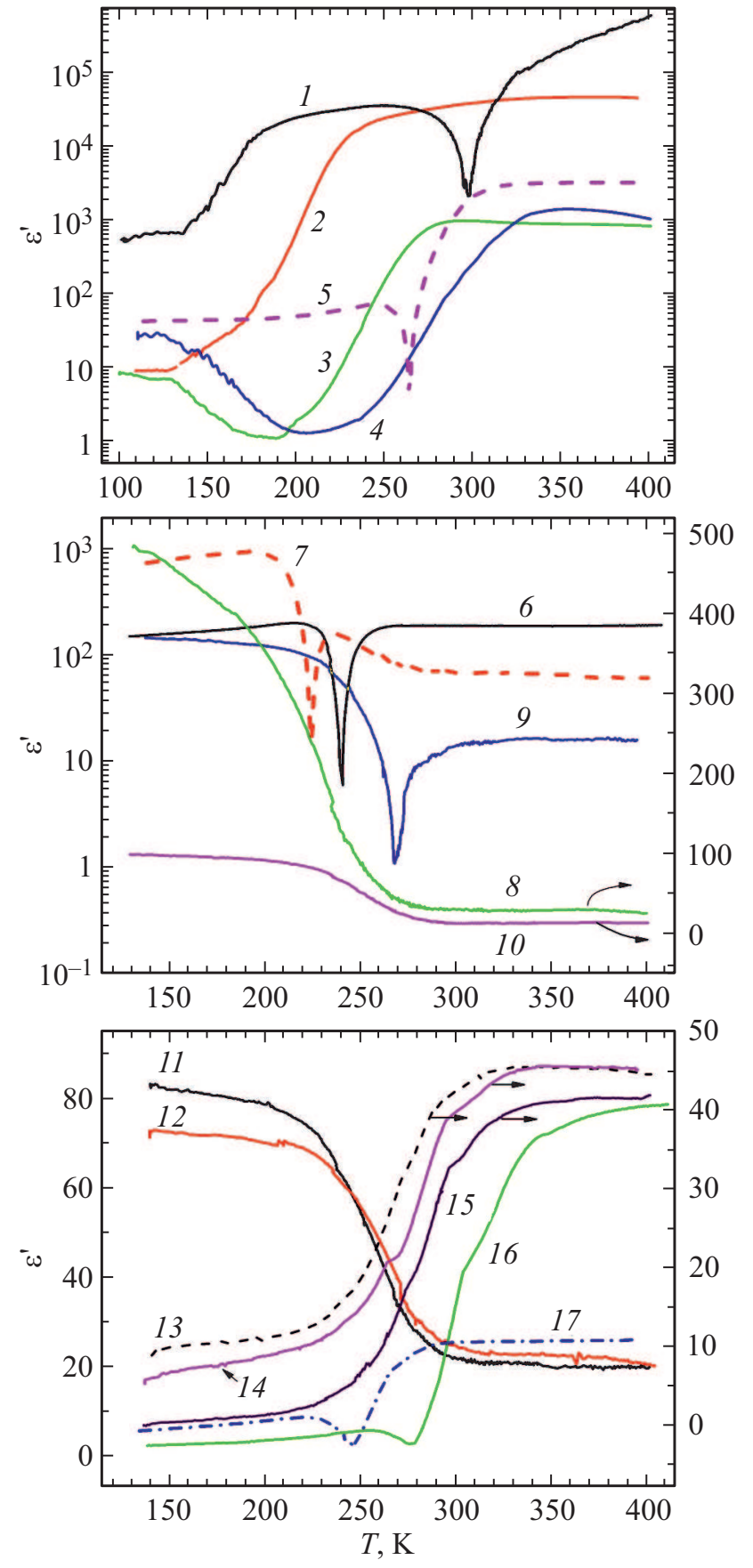

Рис. 1. Температурная зависимость диэлектрической проницаемости $\mathrm{LuFe}_{2} \mathrm{O}_{4}$ при разных $а с$-частотах измерительного напряжения. $1-20 \mathrm{~Hz} ; 2-5 \mathrm{kHz} ; 3-50 \mathrm{kHz} ; 4-$ $500 \mathrm{kHz} ; 5-5 \mathrm{MHz} ; 6-30 \mathrm{MHz} ; 7-35 \mathrm{MHz} ; 8-40 \mathrm{MHz}$; $9-45 \mathrm{MHz} ; 10-50 \mathrm{MHz} ; 11-55 \mathrm{MHz} ; 12-60 \mathrm{MHz}$; $13-65 \mathrm{MHz} ; 14-70 \mathrm{MHz} ; 15-75 \mathrm{MHz} ; 16-100 \mathrm{MHz}$; $17-120 \mathrm{MHz}$.

разность между высоким $T_{H}$ и низким $T_{L}$ значением температуры на полувысоте максимума $\sigma(T)$ (см. таблицу). Минимум $\varepsilon^{\prime}(T)$ для $20 \mathrm{~Hz}$ и $45 \mathrm{MHz}$ совпадает с вершиной кривизны $\sigma(T)$, где резко увеличивается ее наклон $\sim 297 \mathrm{~K}$ и с изломом $\sigma(T) \sim 275 \mathrm{~K}$ для 



Рис. 2. Температурная зависимость удельной электрической проводимости $\mathrm{LuFe}_{2} \mathrm{O}_{4}$ при разных $а с$-частотах измерительного напряжения. $1-20 \mathrm{~Hz} ; 2-5 \mathrm{kHz} ; 3-50 \mathrm{kHz} ; 4-$ $500 \mathrm{kHz} ; 5-5 \mathrm{MHz} ; 6-30 \mathrm{MHz} ; 7-35 \mathrm{MHz} ; 8-40 \mathrm{MHz}$; $9-45 \mathrm{MHz} ; 10-50 \mathrm{MHz} ; 11-55 \mathrm{MHz} ; 12-60 \mathrm{MHz}$; $13-65 \mathrm{MHz} ; 14-70 \mathrm{MHz} ; 15-75 \mathrm{MHz} ; 16-80 \mathrm{MHz}$; $17-100 \mathrm{MHz} ; 18-120 \mathrm{MHz}$.

соответствующих частот. На $\sigma(T)$ для $50 \mathrm{kHz}$ имеются незначительные аномалии вблизи температур $\sim 230$ и $320 \mathrm{~K}$, которые согласно [3] соответствуют температуре магнитного и зарядового упорядочений. С увеличением частоты $a c$ напряжения положения максимумов $\sigma(T)$
Параметры кривых $\varepsilon^{\prime}(T)$ и $\sigma(T)$ при разных ас-частотах

\begin{tabular}{c|c|c|c}
\hline$f, \mathrm{MHz}$ & $\varepsilon^{\prime}(T): T_{\min }, \mathrm{K}$ & $\sigma(T): T_{\max }, \mathrm{K}$ & $\Delta T_{\sigma}=T_{H}-T_{L}, \mathrm{~K}$ \\
\hline $20 \mathrm{~Hz}$ & 297 & - & - \\
$500 \mathrm{kHz}$ & - & 332 & - \\
5 & 265 & 294 & $323-270=53$ \\
30 & 240 & 238 & $256-221=35$ \\
35 & 224 & 215 & $231-196=35$ \\
45 & 270 & - & - \\
40 & - & 173 & - \\
80 & - & 300 & $331-278=53$ \\
100 & 277 & 306 & $343-278=65$ \\
120 & 247 & 254 & $278-230=48$
\end{tabular}

смещаются в низкотемпературную область, а положения минимумов - в обратную сторону. В области частоты от $35 \mathrm{MHz}$ до $40 \mathrm{MHz}$ максимум в $\sigma(T)$ подавляется при этом высокотемпературная ветвь $(T>250 \mathrm{~K})$ нарастает (рис. 2, верхняя и средняя рамки). Дальнейшая эволюция частоты приводит к изменению соотношения низкотемпературной и высокотемпературной ветвей $\sigma(T)$ относительно минимума. При достижении частоты до $100 \mathrm{MHz}$ и выше $\sigma(T)$ вновь приобретает характер выраженного максимума (рис. 2, нижняя рамка).

В результате обобщения большого числа экспериментальных данных [16] установлено, что для ферритов выполняется условие $\varepsilon \propto 1 / \sqrt{\sigma}$, которое объясняет корреляцию между минимумами $\varepsilon^{\prime}(T)$ и максимумами $\sigma(T)$. Частотная зависимость $\varepsilon$ и $\sigma$ для большинства ферритов может быть выражена с помощью релаксационных формул [15]:

$$
\begin{gathered}
\sigma=\sigma_{d}+\frac{\varepsilon_{s}-\sigma_{d}}{1+\omega^{2} \tau^{2}} \\
\varepsilon^{\prime}=\varepsilon_{d}+\frac{\varepsilon_{s}-\varepsilon_{d}}{1+\omega^{2} \tau^{2}}
\end{gathered}
$$

значки $s$ и $d$ соответствуют значениям при очень низкой, и высокой частотах. Время релаксации $\tau$ представляет собой характеристическую временную постоянную феррита. В формуле (1) при увеличении $\omega$ в области $\omega \tau \ll 1$ числитель дроби растет быстрее, чем знаменатель соответственно $d \sigma / d \omega$ положительна, а для (2) ситуация обратная (см. [16] с. 304). Температурная зависимость выражений (1) и (2) при фиксированной частоте определяется зависимостью $\tau=f(T)$, которая, с учетом влияния времени релаксации заполнения глубокого уровня, имеет немонотонный характер [14,17].

Наличие максимума на температурной зависимости $a c$-проводимости является свидетельством термостимулированного опустошения центров захвата (глубокие уровни) электронов, в качестве которых могут выступать различные структурные дефекты образца [17]. На данный механизм проводимости в $\mathrm{LuFe}_{2} \mathrm{O}_{4}$ указывает Maglione и др. [18], которые объясняют происхождение максимума температурной зависимости тока деполяризации термоопустошением ловушек электронов. Такими 
ловушками в $\mathrm{LuFe}_{2} \mathrm{O}_{4}$ являются анионные вакансии кислорода, возникающие при термообработке образца на одной из технологических стадий его изготовления. $a c$-проводимость, обусловленная термоопустошением глубоких уровней, максимальна при $\omega \tau=1$, где $\tau$ - время релаксации заполнения глубокого уровня. Поскольку $\tau$ уменьшается с увеличением температуры $(\tau \propto \exp (\Delta E / k T), \Delta E-$ энергия активации электрона), то положение максимума $\sigma(T)$ должно смещаться в сторону высоких температур при увеличении частоты внешнего напряжения [17]. В нашем случае наблюдается обратная картина (рис. 2, верхняя рамка): с увеличением частоты $a c$-напряжения максимум $\sigma(T)$ сдвигается в сторону низких температур. Следовательно, максимумы $\sigma(T)$ на рис. 2 имеют другую физическую природу.

Проводимость в $\mathrm{LuFe}_{2} \mathrm{O}_{4}$ характеризуется прыжковым механизмом ниже $\sim 320 \mathrm{~K}[9,11]$. Логично предположить, что максимум на $\sigma(T)$ возникает, когда частота перескоков электронов между $\mathrm{Fe}^{+2}$ и $\mathrm{Fe}^{+3}$, направленных в сторону электрического поля совпадает с частотой внешнего напряжения, то есть возникает резонансное состояние $(\omega \tau=1)$. С увеличением температуры увеличивается интенсивность тепловых колебаний ионов и, как следствие, уменьшается число перескоков электронов в единицу времени в сторону поля. При понижении температуры до определенного момента уменьшается рассеивающий фактор, связанный с тепловым движением ионов, что приводит к увеличению числа перескоков электронов, участвующих в проводимости, соответственно резонансное состояние $(\omega \tau=1)$ будет достигнуто при более высоких частотах. С дальнейшим понижением температуры увеличивается энергия активации перескоков и проводимость падает. $\Delta E$ оцененная из наклона прямой в координатах $\ln \sigma=f(1 / T)$ для $20 \mathrm{~Hz}$ (рис. 2, вставка) и из наклона прямой $\ln \tau_{m}=f(1 / T)$, составила величину 0.275 и $0.27 \mathrm{eV}$ соответственно, что близко к значению $0.29 \mathrm{eV}$, полученному в [3]. $\tau_{m}$ определялось из условия $\omega \tau=1$, выполняющегося для максимума $\sigma(T)$.

Учитывая имеющийся в литературе диспут [3,9,11] относительно природы высокого значения $\varepsilon$ для $\mathrm{LuFe}_{2} \mathrm{O}_{4}$, нельзя исключать влияние барьера Шоттки в приконтактной области образца на $\sigma(T)$ и $\varepsilon(T)$. Формирование обедненного слоя (барьера Шоттки) на границе образца является релаксационным процессом со временем релаксации $\tau \geq \tau_{\mathrm{M}}, \tau_{\mathrm{M}}$ - максвелловское время релаксации [19]. При наличии обедненного слоя на границе образца его электрофизические свойства будут устанавливаться в зависимости от параметра $-X$, равной отношению обедненной части образца (запирающего слоя) к остальной ее части. Обратное напряжение внешнего переменного поля на барьерной структуре максимально в первую четверть периода тестового сигнала $1 / 4 f$, во вторую четверть периода напряжение уменьшается, а затем оно меняет знак, и формирование запирающего слоя происходит на противоположной границе образца. Когда $1 / 4 f>\tau_{\mathrm{M}}$ логично предположить, что параметр $X$ будет максимальным, но из-за влияния глубоких центров (роль которых могут играть различные структурные дефекты в зернах и на их границах) и медленных поверхностных состояний толщина запирающего слоя не достигает максимального значения. Максимальное значение $X$ будет достигнуто при $1 / 4 f \approx \tau_{\mathrm{M}}$. Рассчитанное значение $\tau_{\mathrm{M}}=\frac{\varepsilon^{\prime}}{\varepsilon^{\prime \prime}} \frac{1}{\omega}$ при температуре $T=T_{\min }=245 \mathrm{~K}$ для $\sigma(T)$, измеренной на частоте $45 \mathrm{MHz}$ (рис. 2, средняя рамка) составило величину $0.53 \cdot 10^{-8} \mathrm{~s}$, a $1 / 4 f$ для этой частоты равна $0.55 \cdot 10^{-8} \mathrm{~s}$. Как видно из рис. 2, наиболее глубокий минимум $\sigma(T)$ имеет место при частоте $45 \mathrm{MHz}$. При увеличении температуры $\tau_{\mathrm{M}}$ уменьшается, поэтому условие $1 / 4 f \approx \tau_{\mathrm{M}}$ возникает для более высоких частот $a c$-напряжения. Этим объясняется сдвиг минимумов $\sigma(T)$ в сторону высоких температур при увеличении $a c$-частоты (рис. 2, средняя рамка).

Изменение знака $\alpha_{\varepsilon}$ с отрицательного на положительный (рис. 1, нижняя рамка) при увеличении $a c$-частоты от $70 \mathrm{MHz}$ и выше, обусловлено проявлением резонансной поляризации по мере приближения частоты внешнего поля к частоте структурных элементов образца (диполи или ионы), которые могут быть резонаторами в области $\sim 10^{8}-10^{10} \mathrm{~Hz}$ (см. [19] с. 410]).

\section{3. Заключение}

Таким образом, исследование температурно-частотной зависимости комплексной диэлектрической проницаемости керамического $\mathrm{LuFe}_{2} \mathrm{O}_{4}$ выявило наличие особенностей в виде смены знака температурного коэффициента диэлектрической проницаемости, а также в виде максимумов и минимумов $a c$-проводимости в области температур $\sim 150-350 \mathrm{~K}$ и частот $\sim 30-120 \mathrm{MHz}$. Данные особенности взаимосвязаны и обусловлены независимыми резонансными механизмами: совпадением частоты измерительного $a c$-напряжения с частотой перескоков электронов при реализации прыжковой проводимости; близостью периода измерительного сигнала к времени формирования барьерного слоя на границе контакта с образцом; приближением ас-частоты внешнего поля к частоте структурных элементов образца (диполи или ионы). Полученная информация может быть полезной при конструировании функциональных устройств на базе $\mathrm{LuFe}_{2} \mathrm{O}_{4}$.

\section{Финансирование работы}

Работа выполнена в рамках государственного задания Миннауки ВО России (тема: AАAA-A17117021310366-5).

\section{Конфликт интересов}

Авторы не имеют конфликта интересов. 


\section{Список литературы}

[1] N.A. Hill. J. Phys. Chem. B 104, 6694 (2000).

[2] A.P. Pyatakov, A.K. Zvezdin. Phys. Usp. 182, 593 (2012).

[3] N. Ikeda, H. Ohsumi, K. Ohwada, K. Ishii, T. Inami, K. Kakurai, Y. Murakami, K. Yoshii, S. Mori, Y. Horibe, H. Kito. Nature 436, 1136 (2005).

[4] S.V. Sheong, M. Mostovoy. Nature Mater. 6, 13 (2007).

[5] Y.B. Kudasov, D.A. Maslov. Phys. Rev. B 86, 214427 (2012).

[6] J. Van den Brink, D.I. Khomskii. J. Phys. Condens. Matter 20, 434217 (2008).

[7] D.S.F. Viana, R.A.M. Gotardo, L.F. Cotica, M. Ozon-Dionysio, D. Garcia, T.A. Eiras. A.A. Coelho. J. Appl. Phys. 110, 034108 (2011).

[8] Y. Hou, Y. Yao, S. Dong, X. Huang, X. Sun, X. Li. J. Mater. Res. 27, 6, 922 (2012).

[9] S. Lafuerza, J. Garcia, G. Subias, T. Blasco, K. Conder, E. Pomjakushina. Phys. Rev. B 88, 085130 (2013).

[10] Yu.B. Kudasov, M. Markelova, D.A. Maslov, V.V. Platonov, O.M. Surdin, A. Kaul. Phys. lett. A 380, 3932 (2016).

[11] D. Nierman, F. Waschkowski, J. de Groot, M. Angst, J. Hemberger. Phys. Rev. Lett. 109, 016405 (2012).

[12] A. Ruff, S. Krohns, F. Schrettle, V. Tsurkan, P. Lunkenheimer, A. Loidl. Eur. Phys. J., B 85, 290 (2012).

[13] A.G. Gamzatov, G.M. Gajiev, R.A. Aliev, L.L. Emiraslanova, A.R. Kaul, M. Markelova, S.-C. Yu. Appl. Phys. Lett. 112, 092902 (2018).

[14] Р.А. Алиев, А.Г. Гамзатов, Г.М. Гаджиев, Н.С. Абакарова, А.Р. Кауль, М. Маркелова, Л.Л. Эмирасланова. ФТТ 80, 1062 (2018).

[15] A.G. Gamzatov, A.M. Aliev, M.N. Markelova, N.A. Burunova, A.S. Semisalova, N.S. Perov. Phys. Solid State 58, 1143 (2016).

[16] J. Smit, H.P.J. Wijn. Ferrites. Phylips Technical Library (1959).

[17] Л.С. Берман, А.А. Лебедев. Емкостная спектроскопия глубоких центров в полупроводниках. Наука, Л. (1981). C. 28.

[18] M. Maglione, M.A. Subramanian. Appl. Phys. Lett. 93, 032902 (2008).

[19] П.Т. Орешкин. Физика полупроводников и диэлектриков. Высш. шк., М. (1977). 448 с.

Редактор К.В. Емцев 